\title{
The impact of commuters' psychological feelings due to delay on perceived quality of a rail transport
}

Mahdi Rezapour $^{1 凶} \&$ F. Richard Ferraro ${ }^{2}$

Public transport's delay has become an integral part of the daily routine for many commuters, and become more intense as people are under more time pressure and deadline. This study was set forward to study a relationship between various psychological feelings that the commuters might experience due to delay and the level of transport satisfaction. One of the most notorious rail transport in Malaysia was employed as the case study. The passengers of that rail transport often experience more than an hour of delay due to malfunctions or other mechanical problems of that transport. Our instruments include 396 fully completed commuters' responses at the locations of the waiting time. The instruments include three subsections: demographic, physical, and psychological behaviors that commuters might experience. An indicator was also used to obtain the passengers' opinions about the impacts of delay on the level of the perceived quality of the transport. The ordered mixed logit model was employed for the analysis. The mixed model was considered to provide more flexibility and to prevent biased point estimates. That is especially important as it is expected that the point estimates of some commuters' feelings might vary across population based on factors such as the commuters' objectives of travel, or level of urgency to reach the destination. The results highlighted, for instance, the feelings of being tired or nervous to be random in predicting the perceived quality of the transport. The results also indicated that the interactions between age, gender, and level of education, and also neck pain, and an increased heartbeat should be considered, instead of their sole main effects. Also, evaluating the heterogeneity in taste revealed that the change in a random parameter of feeling nervous is dependent on the parameter of commuters' gender. Overall, the results provide important insights regarding various feelings that the commuters experienced, which impact their perceived quality of rail transportation. Extensive discussion regarding the link between the study's findings and mindsponse theory was given in the "Discussion" and "Conclusion" sections of this study.

\footnotetext{
${ }^{1}$ Optim, Mashhad, Iran. ${ }^{2}$ Department of Psychology, University of North Dakota, Grand Forks, ND, USA. 凶email: Rezapour2088@yahoo.com
} 


\section{Introduction}

ublic transportation systems play a vital role in reducing traffic congestion, gasoline consumption, carbon emission, and consequently creating cleaner air (Mees, 2000). Thus, extensive efforts have been made by public transport planners to enhance the satisfaction of public transport. The higher satisfaction could be achieved, for instance, by an improvement in the quality of a certain service, which could lead to greater use of public transport. One of the aspects of public transport, which received much attention, is the delay. Delay is part of waiting time and can be defined as the difference between the actual arrival of public transportation and the expectation of a commuter. The expectation of commuters, on the other hand, might be gained by time schedule presented by policymakers or real-time information display. However, if none of them exist, or no precise information is provided, it could be gained from the experience of a commuter about the average arrival or departure of the public transportation. In addition, delay by some studies has been defined as the proportion of time that a transport does not arrive or depart from a station more than a certain time in minutes; for instance, the arrival of the train more than 5 min in most European countries would be considered late, while in the Netherlands this time is reduced to $3 \mathrm{~min}$, and in Japan this time even defined as 10-15 s (Yuan, 2006).

Delays are a major reason why commuters would be dissuaded from using public transportation. Even it has been shown that transport stressors such as traffic congestion would result in serious implications such as motivational deficiency (Evans et al., 2007). Also, the commuting unreliability and delays have been shown to result in low efficiency in tired workers and cost in London at least 230 million pounds per annum (Cox et al., 2006). As the result, railway companies and policymakers exert their efforts to avoid delays and improve punctuality. The less amount of delay commuters face, the more tendency is expected for commuters to choose that service instead of other modes of transport (Jansson, 1993). However, sometimes delay is inevitable so it is important to know how the delay is translated to the commuters in terms of various psychological behaviors: It is expected that the impact of delay on the commuters' perceived quality of service to be translated from emotional or physical behaviors that the commuter might experience. Understanding those feelings would not only inspire the companies to prevent those feeling but also could help them to find a solution to improve their services, even if they cannot reduce the amount of delay.

In this research, delay acts as a stressor that results in stress and other psychological impacts on commuters, so it is necessary here to clarify exactly what is meant by stress and stressor. According to a definition provided by Lazarus (1998), stress could be defined as a set of psychological and physiological reactions to noxious agents, where the stressor is denoted as the agent, and stress is denoted by the reaction. The underlying reason for stress or other related psychological impacts could occur when the environment requests an individual more than the capacity and resource (Lazarus and Folkman, 1984); which also could be linked to the underlying reasons behind the negative impacts of delay on commuters.

\section{Literature review}

The main frameworks of this study could be divided into three topics. The first part of this study could be looked at from, I) the relationship between the feelings that commuters would experience due to shortcomings of the transport; II) the relationship between commuters' satisfactions and the transport reliability, and III) commuters' behaviors and its relationships with commuters' feelings. All these sections would highlight the plausible relationship between various links in our study being investigated in the literature review.

Commuters' negative feelings due to transports shortcomings. An instruments survey was conducted to evaluate the passengers' waiting behavior in terms of irritation levels due to waiting time at bus stops (Ohmori et al., 2004). The results highlighted that there is a higher irritation level associated with a longer waiting time. In another study, crowding, delay, and accessibility to a railway station were some of the sources of the commuters' anxiety (Cheng, 2010). In that study, it was found that various feelings of anxiety or stress might be due to reaching late to the destination.

So, it is expected for commuters to experience negative feelings due to the shortcomings of public transport.

Importance of the transport's reliability on commuters' satisfaction. Factors impacting public transport commuting satisfaction were evaluated (Cantwell et al., 2009). The results highlighted that the level of satisfaction for public transport users would decrease for those who travel on crowded and unreliable services and those having long waiting times. So, it is expected to have a link between the level of satisfaction and the shortcomings of delay.

Relationship between commuters' feelings and commuters' behaviors. On the other hand, it has been highlighted that the commuter's choice would be varied based on various environmental and conditions of commuters. The relationship between psychological and situational factors on the commuter mode choice was evaluated (Collins and Chambers, 2005). The results highlighted that individual's value on commuting behaviors would be impacted by the environmental threat of cars and also commuting behaviors would be influenced by commuters' consideration of future consequences, and finally time and costs contribute to the commuters' choices. It has been also discussed that understanding the psychological and situational factors would impact the commuter-transport choice and thus understanding those factors is important for shifting the commuters from cars to public transport (Collins and Chambers, 2005).

Based on the above discussion it is reasonable to believe that there are various psychological impacts due to delay, and those feelings are expected to impact the perceived quality of the transport for commuters, and their future behaviors. Also, it is expected for commuters to be varied based on various demographic or environmental characters, so it is important to account for that heterogeneity by considering random parameters model.

Despite the public transport importance, very limited studies have tried to link the psychological feelings that the commuters might experience and satisfaction with rail transport. Also, regarding potential policy and practical implications, it is important to have a clear vision regarding the impacts of the public transport shortcoming on the feelings of the commuters. Malfunctioned and bad experiences of transport are very common in transition economies, especially in developing countries so a better understanding regarding the psychological impact of the public transport shortcoming would expand our understanding to resolve the issue.

\section{Research questions}

Based on the above discussion, it is expected that delay has a negative impact on the perceived quality of public transport. 
However, it is not clear what characteristics of delay might impact the perceived quality of service! The impact of delay could be seen from various emotional or physical feelings that the commuters experience. Thus, the impacts of delay on the perceived quality could also be looked at from those commuters' behaviors. Thus, the study is conducted to understand those impacts, and set forward to answer the specific questions as follows:

- What feelings of the commuters impact rail transport satisfaction?

- How various demographics of the commuters would impact that satisfaction, and are there any interaction terms across those factors?

- Are all commuters react to the delay similarly or there is heterogeneity across those individuals? That is especially important as it has been found that a significant proportion of individual's belief's variance would be influenced by their environmental beliefs (Schultz and Zelezny, 1999). Also, there would be a difference whether commuters with flextime or those without (Lucas and Heady, 2002)

- Is the means of the random parameters' distributions are consistent across all individuals or varies based on some specific observed characteristics?

\section{Data and method}

This section would be presented in two subsections. The first subsection would present the method used in this study and the algorithm for model' parameters estimations, while the second section would talk about the instruments.

Method. In this study, the mixed ordinal logit model was used due to the ordered nature of the response. Also, it was noted that the standard regression models are problematic when the response is in an ordinal scale as the usual assumption for regression are not generally met (McKelvey and Zavoina, 1975). The following paragraphs would outline the formulation of the mixed model, and then it would discuss the implementation of the mixed ordinal logit model in the next subsection. All the processes were implemented in $\mathrm{R}$, and the process is straightforward following the equations and matrices' preparations.

In a random parameter or mixed model, random regression parameters would vary across individual observations $n$ based on some distribution. For a random parameter, the probability of individual $n$ choosing $y$ with a density of $g(\beta \mid \theta)$, with parameter $\theta$, could be written as follows:

$$
P_{n}\left(y_{n} \mid \theta\right)=\int_{\beta} P_{n}\left(y_{n} \mid \beta\right) g(\beta \mid \theta) \mathrm{d} \beta
$$

where the density of $\beta$ is $g(\beta \mid \theta)$. As the integral does not have a close form, we could assume, for instance, the normal distribution for random parameters with mean and variance of $\mu$ and $\Sigma$, respectively. $\beta_{i} \sim N(\mu, \Sigma)$, where $\beta_{i}=\mu+\Sigma^{-0.5} v_{i}$, for $v_{i} \sim N(0, I)$, so we have

$$
P_{n}\left(y_{n} \mid \theta\right)=\int_{-\infty}^{\infty} P_{n}\left(y_{n} \mid \theta\right) \frac{1}{\sqrt{2 \pi}} \exp \left(-0.5 \vartheta^{2}\right) \mathrm{d} \vartheta
$$

As the above integral also does not have a closed-form solution with a method such as maximum-likelihood (ML) estimation, by using a good approximation for $g(\beta \mid \theta)$ the integral could be solved. That is through random draws from distribution.

For the analysis, both thresholds and some coefficients might vary across the observations. The model contains $J-n$ threshold parameters, where $n$ is the number of response categories, with marginal utility coefficients of $\beta$.
The ordinal logit model could be defined as latent regression approach as follows (Bhat and Pulugurta, 1998):

$$
C_{i}=k \text { only if } \psi_{k-1}<C_{i}^{*} \leq \psi_{k}, k=0,1, \ldots, K, \psi_{k-1}=-\infty, \psi_{k}=\infty
$$

where $C_{i}^{*}$ represents the latent class, and $\Psi_{k-1}$ and $\Psi_{k}$ are thresholds at both ends of the distribution. The probability associated with outcomes for ordinal response could be written as

$$
\begin{aligned}
\operatorname{Prob}\left[y_{i}\right. & \left.=j \mid x_{i}\right]=\operatorname{Prob}\left[\psi_{j}-\beta^{\prime} x_{i}\right]-\operatorname{Prob}\left[\psi_{j-1}-\beta^{\prime} x_{i}\right], \\
j & =0,1, . ., J
\end{aligned}
$$

From the above equation, it should be noted that $\beta^{\prime} x_{i}$ would be used as a representative for latent regression with the response of $y_{i}^{*}$. The following assumptions need to be made (Greene and Hensher, 2010): to preserve positive signs for all probability we need to have $\Psi_{j}>\Psi_{j-1}$, also to have the support to be entirely real number $\Psi_{1}=-\infty$, and $\Psi_{j}=\infty$. In addition, we set the variance as $\operatorname{var}\left[\varepsilon_{i} \mid x_{i}\right]=\pi^{3} / 3$. From the above equation, for instance, based on the cumulative density function (CMF) for $y=1$ we would have: $\operatorname{Prob}\left[y_{i}=1 \mid x_{i}\right]=F\left(0-\beta^{\prime} x_{i}\right)-F\left(-\infty-\beta^{\prime} x_{i}\right)=F\left(-\beta^{\prime} x_{i}\right)$. Now, the maximum likelihood could be used for maximization Eq. (4), or log-likelihood (LL), as follows (Greene, 2003):

$$
\begin{aligned}
\mathrm{LL} & =\sum_{i=1}^{n} \sum_{j=0}^{J} m_{i j} \log \left[F\left(\psi_{j}-\beta^{\prime} x_{i}\right)-F\left(\psi_{j-1}-\beta^{\prime} x_{i}\right)\right] \\
& =\sum_{i=1}^{n} \sum_{j=0}^{J} m_{i j} \log \left[F_{i, j}-F_{i, j-1}\right]
\end{aligned}
$$

where $m_{i j}=1$ for $y_{i}=j$, and 0 otherwise.

It should be noted that the threshold parameters $\Psi_{k}$ in the above equations have been treated as nuisance parameters, which would be used for computation purposes. However, it has been argued in the literature review that in the psychology application if people are in strong agreement or disagreement, the cut point is expected to be tightly bunched in the middle of the distribution, while when the people are not willing to express their strong feelings, the point is expected to be more widely dispersed (Daykin and Moffatt, 2002).

Without considering the threshold the model could become inconsistent, and the estimations would be impossible. As we are dealing with simulated random draws, the maximum likelihood often is called simulated likelihood estimation. The "Model parameters estimation" section discusses the implemented method of the ordered mixed logit model with correlated random parameters. We considered both correlated and non-correlated random parameters due to similarity in the goodness of fit. However, with a small modifications, the correlation across random parameters would be ignored to come up with the standard ordered mixed model.

Model parameters estimation. It is worthy to discuss how the parameters are estimated. Again, as the difference between the syntaxes of accounting and not accounting for correlation across random parameters a were similar in most parts, this section would discuss considered correlation across random parameters.

1. Get the random and fixed parameters.

2. Identify the categories of the response by considering the unique categories. As, in this study, no respondent chose the last category of the considered dependent variable, we have only four categories.

3. A matrix would be created by $J-2$ columns, and rows to the number of observations.

4. Initial values would be given to the threshold parameters: threshold 1 and 2. As we have four categories for our 
response, we would have $J-2$ or 2 values for thresholds being saved as alpha.

5. There would be few more thresholds, which should be aggregated by the above threshold. Those thresholds would not be updated and varied but would be considered in the model. Those are -inf and +inf.

6. Fixed parameters would be saved as gamma.

7. As here we would consider correlated random parameters, the process of creating random parameters would be presented as $\beta_{i r}=\beta+L \omega_{i r}$, where $L$ is the lower triangular producing the covariate matrix of random parameters, and $\omega_{i r}$ would be $r$ draws for $i$ observation. For instance, by having two random parameters, there would be three values: SDs of the parameters and their correlations. That result would be a lower triangular matrix of 2 by 2 with initial values of 0.1 . Now based on the above equation the cross product of the random numbers for the first observation and lower triangular would be computed and would be added to the initial value of $\beta$. As we also have heterogeneity in taste for some of the random parameters, for that predictor there would be heterogeneity based on $\beta_{i r}=\beta+$ $\pi s_{i}+L \omega_{i r}$. In other words, the resultant value of above as $\beta_{i r}$, one more time would be added by $\pi s_{i}$. Where the fixed effect of the parameters is $\pi$, and the initial values of the heterogeneity is $s_{i}$.

8. The cross-product of initial values of fixed parameters and their vectors would be saved as $Z B$.

9. Now, the two items, $\left(\beta_{i r} . X_{b}\right)$ and fixed effects (ZB), would be summed up, integral, and the maximum likelihood would be conducted on the sum values of the two: index $=$ $x b+Z Z$.

10. Now moving to Eq. (5). First $T=$ threshold $[y+1]$-index and $T T=$ threshold $[y]$-index would be created.

11. Using the cumulative density function (CMF) for the above equation, $F(T)-F(T T)$, and dividing by $R$ would be saved as $\log$-likelihood $P_{i}=\frac{1}{R} \sum_{r=1}^{R} P_{i r}$.

12. Maximum-likelihood function based on Gradient and Hessian would be employed to find the optimum values of the parameters maximizing the log-likelihood.

The instruments. A total of 396 fully answered responses were collected and considered for the analysis. Most of the respondents were female, college students, having a higher education of at least a diploma, means of 0.530 and 1.7 for education and gender, respectively (see Table 1). Also, most of the respondents belong to the age group of $15-25$, mean of 0.162 . In sections $\mathrm{B}-\mathrm{D}$ the respondents were asked on 5-scale Likert question, where the scale had the following alternatives: strongly agree (as 1), agree (2), undecided (3), disagree (4), strongly disagree (5). For instance, based on Table 1, feeling tired and disgust were some of the feelings that respondents agree the most to experience while experiencing delay for that type of rail transport. The initial examination of the data reveals that as the expected overwhelming majority of the respondent rated the impact of delay very negatively and in favor of various emotional or physical feelings.

In the instruments, the travelers were asked to indicate to what extent they agree they experience various emotional or physical feelings while facing the delay of the train. On the same scale, they were asked how much they agree that delay would impact the quality of the rail transport. So various feelings were used as a proxy for the delay, and then those feelings were used as predictors for the degree of belief about the impacts of delay on the quality of the rail transport.

\begin{tabular}{|c|c|c|c|c|}
\hline Variables & Average & Variance & Min & Max \\
\hline \multicolumn{5}{|l|}{ Response } \\
\hline $\begin{array}{l}\text { D5, the delay has a negative } \\
\text { impact on the quality of KTM }\end{array}$ & 1.490 & 0.504 & 1 & 4 \\
\hline $\begin{array}{l}\text { Predictors } \\
\quad \text { Individual attributes }\end{array}$ & \multicolumn{3}{|c|}{ Predictors } & \\
\hline $\begin{array}{l}\text { Education, higher than Diploma as } \\
0 \text {, others as } 1\end{array}$ & \multicolumn{3}{|c|}{0 , others as 1} & 1 \\
\hline Age, $18 \leq 25$ as 0 , others as 1 & 0.162 & 0.136 & 0 & 1 \\
\hline A1, Gender, male as 1, female as 2 & 1.758 & 0.184 & 1 & 2 \\
\hline \multicolumn{5}{|l|}{ Physical feelings } \\
\hline C1, Neck pain & 2.306 & 1.292 & 1 & 5 \\
\hline C4, muscle stiffness & 2.230 & 1.220 & 1 & 5 \\
\hline $\mathrm{C} 5$, increased heartbeat & 2.910 & 1.293 & 1 & 5 \\
\hline C11, drawing sensation in body & 1.977 & 1.060 & 1 & 5 \\
\hline $\mathrm{C} 14$, feel nervous & 3.126 & 5.91 & 1 & 5 \\
\hline $\mathrm{C} 7$, feel tired & 1.871 & 0.892 & 1 & 5 \\
\hline \multicolumn{5}{|l|}{ Psychological feelings } \\
\hline B3, Disgust & 1.873 & 0.971 & 1 & 5 \\
\hline B4, feel anxious & 1.992 & 1.040 & 1 & 5 \\
\hline
\end{tabular}

It was hypothesized in this study that the direct questions of the impact of various feelings on the quality of the rail transport would result in possible arbitrary responses of the commuters, and even leaving those options blank. One of the reasons could be due to the hardship of questioning the impacts of various feelings on the quality of rail transport. Although indirect questionings of instruments have been extensively used in other social studies, this study used indirect questioning due to preventing imposition of hardship in answering questions on the respondents. Indirect questioning has been used extensively in the literature. For instance, an indirect question was used to detect intimate partner violence in an instrument's application (Fulfer et al., 2007).

In this study, the instruments were distributed at the station of Serdandg, which is one of the main stations of Keretapi Tanah Melayu (KTM). The surveys were distributed during off-peak hours from 4 to 7 p.m. to be consistent in our evaluation. Instruments were translated into the local language, Malay, by a Malaysian Ph.D. student in the field of education. The instruments had an introduction explaining the objective of the study, and various sections of the instruments. The respondents were requested to leave the instruments blank if they are not interested. It should be noted that no random sampling was implemented in this study.

The instruments had 4 parts: background (9 questions), psychological effects (4 questions), physical effects (14 questions), and general questions (5 questions). All the questions except for the first part were based on a 5-scale question type. It was noted that due to the satisfying behavior of respondents, some of the responses would result in incomplete or biased information retrieval (e.g. choosing the first response alternative) or no information retrieval (Krosnick, 1991). A solution has been proposed by giving an alternative of "I do not know" or "Undecided" instead of reporting an opinion. As a result, we incorporated in our instruments an alternative of "undecided". An undecided answers could be considered as a middle response (Groothuis and Whitehead, 2002). To evaluate the feelings that the commuters might feel due to delay, the questions were presented to the respondent like "I feel angry when I face a delay of KTM" and the respondent would answer on a scale of 1-5, from 1-strongly agree to 5-strongly disagree. 


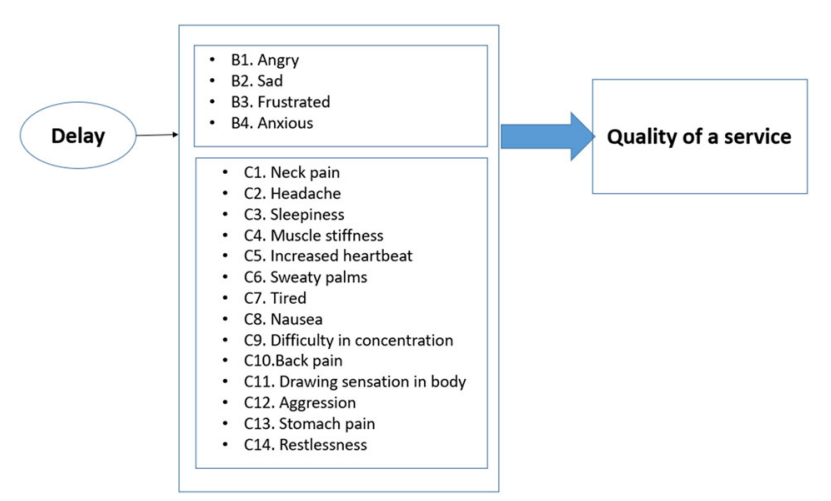

Fig. 1 List of all questions included in parts B and C of the instruments.

A: psychological feelings that commuters might experience due to delay, B: physical feelings that commuters might experience, quality of a service: would be the response.

The physical section of the instruments was based on the Cohen-Hoberman inventory of physical symptoms (CHIPS) (Cohen and Hoberman, 1983). That is a list of 39 common physical symptoms highlighting the relationship between negative life stress, and various physical symptomatology. Those include factors such as back pain, diarrhea, and headache. The factors were filtered to only 14 due to the expected impacts of delay based on those feelings, see $\mathrm{C}$ part of Fig. 1.

On the other hand, various sources were used for the design of psychological aspects of instruments. For instance, a self-report measure of stress was developed and tested (Greller and Parsons, 1988). The scale includes various physiological and psychological descriptors. Psychological factors such as being angry, nervous, and stress. Also, some of the physical factors included a stiff neck and feeling tired. A study was conducted to illustrate the capability of a cognitive-motivational-relational theory for predicting emotions (Lazarus, 1991). In that study, 15 different emotions were identified including negative emotions such as anger, anxiety, sadness, and disgust. We implemented a similar feeling for section, B part of Fig. 1 .

Again, one of the reasons behind the indirect connection was an associated difficulty of directly connecting various feelings to the quality of the transport. For instance, it might be awkward or challenging to ask the respondents, for instance, "feeling of a headache due to delay negatively impact the quality of rail transport". So, first, we translated the impact of delay into various emotional and physical feelings, and then their transformed effects were linked to the quality of the transport. All included feelings of the instrument in sections $C$ and B are included in Fig. 1.

Finally, it should be noted that another study in the literature review used a similar case study, which we used in the current manuscript. Rail passenger crowding was evaluated with the help of factor analysis and structural equation modeling (SEM) (Mohd Mahudin et al., 2012). The study came up with an instrument to capture crowding and links it with an experience of feeling stress and exhaustion. Feeling irritable, stressed, frustrated, tense, and unpleasant were some of the factors that were considered in that study.

\section{Results}

All the psychological aspects being considered in this study are based on the recommended feelings, which could be due to stress, in the literature. After identifying the feelings based on delay, a question in the last section of the questionnaire also asked to see how that delay impacts the quality of the KTM. Our dependent variable is the perceived impact of delay on the quality of KTM, and our explanatory variables are various psychological feelings and demographic characteristics that the commuters experience while experiencing a delay.

Out of the incorporated variables (see Fig. 1), six physical (neck pain, muscle stiffness, increased heartbeat, drawing sensation in the body, feeling nervous and feeling tired), and two psychological factors (feeling disgusted and anxiety) were found to be important in the prediction of the perceived quality of KTM due to delay (see Table 2). In addition, all interaction terms, and initially the possibility of all variables to be random were considered in this study. Accounting for interaction is important as in case of the presence of correlation terms, not considering those terms, and interpreting just the main effects, would result in erroneous interpretation of the main effects. In other words, the individual main effects cannot and should not be considered without considering their interaction terms.

As discussed in a previous section, due to almost similar process for estimating the standard mixed ordinal logit model, and the model considered correlation across random parameters, the results of both models are presented in Table 2. In other words, and based on the previous subsection description while for correlated random parameters we have $\beta_{i r}=\beta+L \omega_{i r}$, the value would be changed for the standard model to $\beta_{i r}=\beta+\sigma \omega_{i r}$, where $\sigma$ accommodate standard deviation of a random parameter. Also, the goodness of fit based on Akaike information criterion (AIC) is almost identical for both models so due to simplicity of the standard random model and a slight improvement in model fit, the discussion would be based on model A, non-correlated random parameter.

Interaction terms. The subsection would answer the questions of "Are various commuters with different demographic characteristics perform similarly in response to delay and sense of satisfaction?" or "Are their significant interaction terms between commuters with different characteristics?"

Various personal characteristics such as age, and personality type play a major role in explaining the source of stress level (Barling et al., 2004). So, we considered various demographic characteristics in our instruments. As can be seen from Table 2, there is a significant three-term interaction term between age, gender, and education. For interpretation of the model, all main effects, two-term interaction terms, and three-term interaction terms should be considered. The interaction is expected, somehow, due to the nature of the study: female commuters in Malaysia are expected to be more educated. The second interaction, which was considered and found to be important is the interaction between $\mathrm{C} 1$ (neck pain) and C5 (increased heartbeat). The results indicate that these two items work together in impacting the perceived quality of rail transport due to delay.

Random parameters. This section is set forward to see if there is a significant heterogeneity across the commuters in response to delay and consequently their perceived satisfaction about the rail transport.

It should be noted that although a slight uncertainty is associated with random parameter of C14 $(p$-value $=0.1)$, the results of goodness of fit, AIC, highlighted that inclusion of $\mathrm{C} 14$ as random would result in a slight improvement in the model fit (638 for model A, versus 639 for not considering C14 as random). It should also be mentioned that normal distribution considered for both random parameters. The randomness of variables C7 and $\mathrm{C} 14$ could be related to the fact that there are differences in reacting to delay across individuals (Cleary, 1987). In other words, delay does not always have the same impact on the 
On the other hand, that difference in commuters' perception and view about the circumstances would result in evaluation of delay differently, so there would be varied degree of feelings due to variations in mind interpretations regarding the circumstances: while some commuters having a higher degree of being nervousness or tiredness others have a lower degree of those factors. So, that is why our results highlighted commuters' feeling regrading being nervous and being tired are random in shaping the perceptions regarding the quality of the transport.

On the other hand, as noted by Levy et al. (2007), mindset or believes are expected to be changed based on individuals' characteristics. So, our result about the impact of feeling of tiredness on the perceived quality of delay could be linked to that theory, which showed the impact of feeling of tiredness would be varied based on commuters' gender. In other words, the heterogeneity across commuters' characteristics of tiredness could be modeled based on commuters' characteristics of gender.

Additionally, while there are heterogeneities across commuters' feelings of being nervous or tired, there are more consistency across commuters' feelings of being anxious, disgust, drawing sensation in body, and having muscle stiffness. That highlight regardless of the perceived judgment of the mind about delay, in a similar manner, commuters believe that they have increased feelings of the above while judging the quality of the rail transport.

Finally, it was found that the reaction of the mindset of the commuters in response to the delay could be defined as an interaction term between neck pain and increased heartbeat, instead of their sole main effects. In other words, the commuters' reactions to the delay based on the judgment regarding the circumstances would impact commuters in terms of the neck pain and heartbeat simultaneously. Moving to the three term interaction terms of age, gender, and education. These factors could be considered as types of commuters' characteristics shaping their perception regarding the circumstances and their threshold. The reason behind interaction terms could be due to the fact that, for instance, female commuters are expected to be more educated and younger.

Limitations. The limitations of this study could be summarized as follows. First, this study did not control for equal population subgroups of respondent. For instance, the majority of the respondent are weighted toward female and younger commuters. So, the result should be interpreted by caution. Second due to extreme negative impacts of the transport, some respondents' feelings might persuade them to respond to the questions in a more extreme way. Although that factor could not be directly identified, that could be taken into consideration while interpreting the results. The extreme responses could be observed from the descriptive statistics of the responses being skewed to the strongly agree.

However, given the unique and extreme conditions of the case study and the fact that we had enough observations for the minority groups, the limitations could be considered as acceptable, but more studies are needed to reinvestigate the findings of this study.

\section{Conclusion}

The findings of this study would provide an opportunity for the policy makers to understand the feelings that the commuters might experience during waiting, and how those feelings would impact their services. It is known that feelings are likely to be induced due to lack of control over the circumstances (Friendly, 2013). Also, the mindset could be changed or adopted to the changing environment (Levy et al., 2007). So even if the policy makers cannot reduce the amount of delay that a service would provide, for instance, by knowing anger, or interaction of neck pain and increased heartbeat are the translated impact of delay on the perceived quality of their services, they could provide an environment for commuters to relieve the impact of that psychological feeling. That might be achieved, for instance, by providing a better environment. Example, in terms of shelter or bench type at the station, for the service so passengers would wait for a rail transport comfortably and with minimal negative psychological feelings.

Based on the identified results, it is found that commuters' reaction to delay is dependent on the specific environmental and demographic characteristics. So, while some commuters react to the shortcoming of transport delay mildly, others react more severely. As discussed, for instance, the three terms interaction terms highlight the relationship between commuters in terms of age, gender, and education. The interaction terms have important implications for policy makers and especially for the future studies to take into account those terms. For instance, the three terms interaction terms highlighted that especially the negative feeling of delay is aggregated for higher-age commuters. Thus more efforts are needed to reach out to the group at higher risk to provide incentive and more information regarding the condition of the rail transport. Especially discount might be provided for that special group.

It should be noted that based on the mindsponge theory, the minds' multi-filtering processes would help individuals overcome the challenge of the information flood by considering only appropriate values and worthy opportunities (Vuong et al., 2014; Vuong and Napier, 2015). So, due to lack of trust between commuters and policy makers, it is likely even by providing the real-time information regarding the arrival of the train, the commuters would filter out the provided information due to mistrust. So, the policy makers should take a realistic and trustworthy step by giving the commuters the trust they need so they can digest and trust the information to adjust their expectation for sustaining less stress, and consequently having a higher satisfaction with the transport.

Finally, the mindset expectation itself would be derived from the individuals' expectations about themselves and others (Goldstein and Brooks, 2007). So, recommendation could also be given to the commuters who frequently experience delay so their expectations could be adjusted. It could be recommended that the commuters should be reminded that mostly they are not in control of certain factors and environment. Accepting that fact would relieve the tremendous psychological and physical impacts of the delay that the commuters would experience. Although that might not seem an optimal scenario, but if there would be no immediate solution to the problem that seems one of the viable solutions.

\section{Data availability}

The data may be provided based upon an appropriate request.

Received: 1 April 2021; Accepted: 14 July 2021;

Published online: 02 August 2021

\section{References}

Barling J, Kelloway EK, Frone MR (2004) Handbook of work stress. Sage publications.

Bhat CR, Pulugurta V (1998) A comparison of two alternative behavioral choice mechanisms for household auto ownership decisions. Transp Res Part B 32 (1):61-75

Cantwell M, Caulfield B, O'Mahony M (2009) Examining the factors that impact public transport commuting satisfaction. J Public Transp 12(2):1 
Cheng Y (2010) Exploring passenger anxiety associated with train travel. Transportation 37(6):875-896

Cleary PD (1987) Gender differences in stress-related disorders. Gender Stress 39-72.

Cohen S, Hoberman HM (1983) Positive events and social supports as buffers of life change stress 1. J Appl Soc Psychol 13(2):99-125

Collins CM, Chambers SM (2005) Psychological and situational influences on commuter-transport-mode choice. Environ Behav 37(5):640-661

Cox T, Houdmont J, Griffiths A (2006) Rail passenger crowding, stress, health and safety in Britain. Transp Res Part A 40(3):244-258

Daykin AR, Moffatt PG (2002) Analyzing ordered responses: a review of the ordered probit model. Underst Stat 1(3):157-166

Evans GW, Brauchle G, Haq A, Stecker R, Wong K, Shapiro E (2007) Young children's environmental attitudes and behaviors. Environ Behav 39(5):635-658

Friendly FW (2013) Due to circumstances beyond our control... Random House.

Fulfer JL, Tyler JJ, Choi NJ, Young JA, Verhulst SJ, Kovach R, Dorsey JK (2007) Using indirect questions to detect intimate partner violence: the SAFE-T questionnaire. J Interpers Violence 22(2):238-249

Goldstein SE, Brooks RB (2007) Understanding and managing children's classroom behavior: creating sustainable, resilient classrooms. John Wiley \& Sons, Inc.

Greene WH (2003) Econometric analysis. Pearson Education.

Greene WH, Hensher DA (2010) Modeling ordered choices: a primer. Cambridge University Press.

Greller M, Parsons CK (1988) Psychosomatic complaints scale of stress: measure development and psychometric properties. Educ Psychol Meas 48(4):1051-1065

Groothuis PA, Whitehead JC (2002) Does don't know mean no? Analysis of don't know'responses in dichotomous choice contingent valuation questions. Appl Econ 34(15):1935-1940

Jansson K (1993) Optimal public transport price and service frequency. J Transp Econ Policy 33-50.

Krosnick JA (1991) Response strategies for coping with the cognitive demands of attitude measures in surveys. Appl Cogn Psychol 5(3):213-236

Lambert E, Straznicky N, Eikelis N, Esler M, Dawood T, Masuo K, Schlaich M, Lambert G (2007) Gender differences in sympathetic nervous activity: influence of body mass and blood pressure. J Hypertens 25(7):1411-1419

Lazarus RS (1998) The stress and coping paradigm. Fifty years of the research and theory of RS Lazarus: an analysis of historical and perennial Issues. pp. $182-220$.

Lazarus RS (1991) Progress on a cognitive-motivational-relational theory of emotion. Am Psychol 46(8):819

Lazarus RS, Folkman S (1984) Stress, appraisal, and coping. Springer publishing company.

Levy O, Beechler S, Taylor S, Boyacigiller NA (2007) What we talk about when we talk about 'global mindset': managerial cognition in multinational corporations. J Int Bus Stud 38(2):231-258

Lucas JL, Heady RB (2002) Flextime commuters and their driver stress, feelings of time urgency, and commute satisfaction. J Bus Psychol 16(4):565-571

McKelvey RD, Zavoina W (1975) A statistical model for the analysis of ordinal level dependent variables. J Math Sociol 4(1):103-120

Mees P (2000) A very public solution: transport in the dispersed city.

Mohd Mahudin ND, Cox T, Griffiths A (2012) Measuring rail passenger crowding: scale development and psychometric properties. Transp Res Part F 15 (1):38-51

Nguyen M (2021) Suicidal ideation and behavior rethinking: the mindsponge approach. OSF Preprints, p. 13.
Ohmori N, Hirano T, Harata N, Ohta K (2004) Passengers' waiting behavior at bus stop. In: Wang KCP, Xiao, G (eds) Proceedings of the second International Conference on Transportation and Traffic Studies, ICTTS 2000, Beijing, China, July 31-August 2, 2000.

Sainsbury P, Gibson JG (1954) Symptoms of anxiety and tension and the accompanying physiological changes in the muscular system. J Neurol Neurosurg Psychiatry 17(3):216

Schultz PW, Zelezny L (1999) Values as predictors of environmental attitudes: evidence for consistency across 14 countries. J Environ Psychol 19(3):255-265

Vuong QH (2016) Global mindset as the integration of emerging socio-cultural values through mindsponge processes. In: Global mindsets: exploration and perspectives. pp. 109-126.

Vuong QH, Napier NK (2015) Acculturation and global mindsponge: an emerging market perspective. Int J Intercult Relat 49:354-367

Vuong QH, Napier NK, Vu H, Nguyen MC, Tran TD (2014) Measuring corporate innovation capacity: experience and implications from i2Metrix implementation in Vietnam. ASEAN J Manag Inf 1(1):1-17

Yuan J (2006) Stochastic modelling of train delays and delay propagation in stations. Eburon Uitgeverij BV.

\section{Competing interests}

The authors declare no competing interests.

\section{Declaration of helsinki}

The survey strictly conforms to the World Medical Association (WMA) Declaration of Helsinki.

\section{Additional information}

Correspondence and requests for materials should be addressed to M.R.

Reprints and permission information is available at http://www.nature.com/reprints

Publisher's note Springer Nature remains neutral with regard to jurisdictional claims in published maps and institutional affiliations.

(c) (i) Open Access This article is licensed under a Creative Commons BY Attribution 4.0 International License, which permits use, sharing adaptation, distribution and reproduction in any medium or format, as long as you give appropriate credit to the original author(s) and the source, provide a link to the Creative Commons license, and indicate if changes were made. The images or other third party material in this article are included in the article's Creative Commons license, unless indicated otherwise in a credit line to the material. If material is not included in the article's Creative Commons license and your intended use is not permitted by statutory regulation or exceeds the permitted use, you will need to obtain permission directly from the copyright holder. To view a copy of this license, visit http://creativecommons.org/ licenses/by/4.0/.

(C) The Author(s) 2021 